\title{
A Note on the Notions of Topological Entropy
}

\author{
Wong Koon Sang ${ }^{1}$ and Zabidin Salleh ${ }^{2, *}$ \\ ${ }^{1}$ School of Informatics and Applied Mathematics, Universiti Malaysia Terengganu, 21030 Kuala Nerus, \\ Terengganu, Malaysia; e-mail: kswong0910@gmail.com \\ ${ }^{2}$ School of Informatics and Applied Mathematics, Universiti Malaysia Terengganu, 21030 Kuala Nerus, \\ Terengganu, Malaysia; e-mail: zabidin@umt.edu.my \\ ${ }^{*}$ Corresponding author
}

\begin{abstract}
Topological entropy is used to determine the complexity of a dynamical system. This paper aims to serve as a stepping stone for the study of topological entropy. We review the notions of topological entropy, give an overview on the relation between the notions and fundamental properties of topological entropy. Besides, we cover the topological entropy of the induced hyperspaces and its connection with the original systems. We also provide a summary on the latest research topic related with topological entropy.
\end{abstract}

\section{Introduction}

In the study of dynamical systems, one of the topics is to determine the chaotic behaviour of the systems. There are several methods to determine the complexity of the systems. Topological entropy is one of accepted methods that we use to determine the complexity of the systems. Before the term "entropy" was studied in the aspect of topology and dynamical systems, this term has been widely used in other research fields containing different meanings.

The term "entropy" was formed from two Greek words: en which means "in" and

Received: October 19, 2018; Accepted: October 25, 2018

2010 Mathematics Subject Classification: 54H20, 37B40, 54C70, 54C60.

Keywords and phrases: topological entropy, dynamical systems, induced hyperspaces, set-valued functions.

Copyright () 2019 Wong Koon Sang and Zabidin Salleh. This is an open access article distributed under the Creative Commons Attribution License, which permits unrestricted use, distribution, and reproduction in any medium, provided the original work is properly cited. 
trope which means "transformation". This term has been widely used in various research fields but carried different meanings. In 1864, this term made it first appearance in the field of thermodynamics. Clausius [46] defined the change in entropy of a body as the heat transfer divided by the temperature. Around 1870s, Boltzmann [45, Part III Section 5] introduced entropy in the field of statistical mechanics to calculate the degree of uncertainty about the macrostate of the system that remains if given only the probability vector. Inspired by Boltzmann's entropy, Shannon [38] adopted the term entropy in information theory and defined it as a measure of the average information content associated with a random outcome.

In 1950s, Kolmogorov [25, 26] introduced the concept of entropy in dynamical system as a measure-preserving transformation and studied the attendant property of completely positive entropy (K-property). Later his student Sinai [39] formulated a more general version of entropy which known as Kolmogorov-Sinai entropy that is suitable for arbitrary automorphisms of Lebesgue spaces. Finally in 1965, the concept of the topological entropy was first introduced by Adler et al. [1] for continuous map on a compact topological space. Their idea was heavily inspired from Kolmogorov-Sinai entropy and the relation between these two entropies was proved by Goodwyn [16, 18]. Reader can refer to [21, 47] for extended history of entropy.

After 1965, different notions of topological entropy have been proposed by other researchers $[2,5,6,19,20,33]$. Most of the new notions extended the concept to a more general functions or spaces. Despite of that, the idea of measure the complexity of the systems was preserved among all these new notions. In general, if the topological entropy of a system is positive, then we say that the system is topologically chaotic [44, 50].

In this paper, our main purpose is to review different notions of topological entropy and their properties. In Section 2, we review the construction of different notions of topological entropy. Section 3 will discuss the relations between the notions of topological entropy and some fundamental properties of topological entropy. In Section 4 , we study the topological entropy of the induced hyperspace system and review its connection with the topological entropy of the original system. Finally in Section 5, we give a summary on the recent development of topological entropy. 


\section{Notions of Topological Entropy}

In this section, we study the notions of topological entropy introduced by Adler et al. [1], Bowen [2], Canovas and Rodriguez [6], and Liu et al. [30]. Reader might come across other papers that introduce their own notion of topological entropy, we are not able to cover all of them. We only select the notions which widely cited by other researchers and close related to our interest. Throughout this paper, the pair $(X, f)$ means dynamical system, where $X$ is a topological (or metric) space and $f: X \rightarrow X$ is a self-continuous map of $X$ unless explicitly stated in particular subsection.

\subsection{Topological entropy of continuous map on compact topological space}

Adler et al. [1] were the first who introduce the concept of topological entropy which serve as an invariant under topological conjugacy and analogue of measure theoretic entropy. The notion is defined for continuous mappings on compact topological space in terms of open covers. In this subsection, we always let $X$ be a compact topological space and $f: X \rightarrow X$ be a self-continuous map of $X$.

Definition 2.1. Let $\alpha$ be any open cover of $X$. Since $X$ is compact, we can obtain finite subcovers from the cover $\alpha$. Let $N(\alpha)$ denote as the minimal cardinality of all the subcovers of $\alpha$. The entropy of $\alpha$ is defined as $H(\alpha)=\log N(\alpha)$. Note that $N(\alpha) \geq 1$.

Definition 2.2. If $\alpha, \beta$ are any two open covers of $X$, then their join is defined as $\alpha \vee \beta=\{A \cap B: A \in \alpha, B \in \beta\}$. Similarly the join of any finite collection of open covers of $X$ can be defined as $\bigvee_{i=1}^{n} \alpha_{i}=\left\{\bigcap_{i=1}^{n} A_{i}: A_{i} \in \alpha_{i}\right.$ for $\left.i=1, \ldots, n\right\}$.

Since $X$ is compact and $f$ is continuous, for any open cover $\alpha$ of $X$ the family of $f^{-1}(\alpha)=\left\{f^{-1}(A): A \in \alpha\right\}$ is also an open cover of $X$. Obviously we have $f^{-1}(\alpha \vee \beta)=f^{-1}(\alpha) \vee f^{-1}(\beta)\left[1\right.$, Property 6]. Note that $H\left(f^{-1}(\alpha)\right) \leq H(\alpha)$ and the inequality will become equal when map $f$ is onto [1, Property 7]. Hence, we obtain the following lemma and the proof can be found in [43, Lemma 4.1.1].

Lemma 2.3. For every $\alpha$ open cover of $X$ and $f$ continuous, $\lim _{n \rightarrow \infty} \frac{1}{n} H\left(\mathrm{~V}_{i=0}^{n-1}\right.$ $\left.f^{-i}(\alpha)\right)$ exists and is a non-negative real number. 
Definition 2.4. For a dynamical system $(X, f)$ and $\alpha$ open cover of $X$, we define the topological entropy of $f$ with respect to cover $\alpha$ by

$$
h(f, \alpha)=\lim _{n \rightarrow \infty} \frac{1}{n} H\left(\bigvee_{i=0}^{n-1} f^{-i}(\alpha)\right)
$$

and the topological entropy of $f$ by

$$
h(f)=\sup _{\alpha}\{h(f, \alpha): \alpha \text { open cover of } X\} .
$$

Clearly from Definition 2.1, we can see that $h(f) \geq 0$.

\subsection{Topological entropy of uniformly continuous maps on metric space}

Bowen [2] defines his version of topological entropy for a uniformly continuous map on a metric space. From [15] we know that with Bowen's notion we can relate the topological entropy and the measure theoretic entropy. Note that the metric space is not necessarily compact. In this section, $(X, d)$ means a metric space and $f: X \rightarrow X$ is a member of $U C(X, d)$, where $U C(X, d)$ is the space of all uniformly continuous maps of $(X, d)$.

Definition 2.5. For $(X, d)$ and $f \in U C(X, d)$, let $n$ be a positive integer and $\varepsilon>0$. A new metric $d_{n}$ on $X$ is defined by $d_{n}(x, y)=\max _{0 \leq i \leq n-1} d\left(f^{i}(x), f^{i}(y)\right)$. Let $E \subset X, E$ is $(n, \varepsilon)$-separated set of $X$ if for all $x, y \in E$ where $x \neq y$, the inequalities $d_{n}(x, y)>\varepsilon$ hold. A set $F \subset X$ is said to be $(n, \varepsilon)$-spans $K \subset X$ (with respect to $f$ ) if for all $x \in K$ there exists $y \in F$ such that $d_{n}(x, y) \leq \varepsilon$.

Definition 2.6. Let $K$ be a compact subset of $X, r_{n}(\varepsilon, K)$ is denoted as the smallest cardinality of any subset $F$ that $(n, \varepsilon)$-spans $K$ (with respect to $f$ ) and $s_{n}(\varepsilon, K)$ is denoted as the largest cardinality of any $(n, \varepsilon)$-separated subset of $K$. If we wish to stress the dependence on $f$ we write $r_{n}(\varepsilon, K, f)$ or $s_{n}(\varepsilon, K, f)$ instead. Next, define $r(\varepsilon, K, f)=\limsup _{n \rightarrow \infty} \frac{1}{n} \log r_{n}(\varepsilon, K)$ and $s(\varepsilon, K, f)=\limsup _{n \rightarrow \infty} \frac{1}{n} \log s_{n}(\varepsilon, K)$.

The following lemma is based on Definition 2.5 and Definition 2.6. Reader may refer [2] for detail proof of the lemma. 
Lemma 2.7. Let $K$ be a compact subset of $X$. Then,

(i) $r_{n}(\varepsilon, K) \leq s_{n}(\varepsilon, K) \leq r_{n}\left(\frac{1}{2} \varepsilon, K\right)<\infty$.

(ii) $\varepsilon_{1}<\varepsilon_{2}$ implies $r\left(\varepsilon_{1}, K, f\right) \geq r\left(\varepsilon_{2}, K, f\right)$ and $s\left(\varepsilon_{1}, K, T\right) \geq s\left(\varepsilon_{2}, K, T\right)$.

Definition 2.8. Let $K$ be a compact subset of $X$ and $f$ be a uniformly continuous selfmap of $X$. We define the topological entropy of $f$ with respect to $K$ as

$$
h_{d}(f, K)=\lim _{\varepsilon \rightarrow \infty} r(\varepsilon, K, f)=\lim _{\varepsilon \rightarrow \infty} s(\varepsilon, K, f)
$$

and the topological entropy of $f$ as

$$
h_{d}(f)=\sup _{K}\{h(f, K): K \text { is compact }\} .
$$

Note that in Definition 2.8 we stress the dependence on the metric $d$ used. Later in Section 3 we will see when we restrict $X$ to be a compact metric space, then $h_{d}(f)=h_{d}(f, X)$ and both Adler et al.'s notion and Bowen's notion coincide.

\subsection{Topological entropy for continuous maps on non-compact metric spaces}

Topological entropy has been defined as a measurement of the complexity of a dynamical system. However, Bowen's notion does not fully support this statement. We refer to an example from [51], we have a metric space $(\mathbb{R}, d)$ where $\mathbb{R}$ represents the real line and $d$ is the Euclidean metric and a uniformly continuous map $T: \mathbb{R} \rightarrow \mathbb{R}$ is given by $T(x)=2 x$. It is obvious that the dynamics of this system is simple but $h_{d}(T)=\log 2$. This flaw inspires Canovas and Rodriguez [6] to introduce a new notion of topological entropy modified from the Bowen's notion.

Canovas and Rodriguez's notion is defined for continuous map on metric space. According to [6], this notion is designed to preserve the property of positive topological entropy implies the map has complicated dynamical behaviour, at least for continuous real maps. Additionally this notion preserves some properties that hold for Definition 2.4. Throughout this section, we let $(X, d)$ be a metric space and $f: X \rightarrow X$ be a continuous map. For any set $K \subseteq X$, we can define a new continuous map $\left.f\right|_{K}: K \rightarrow X$ as the restriction of $\operatorname{map} f$ to set $K$.

Definition 2.9. Let $\mathcal{K}(X, f)$ denote as the collection of all compact subsets of $X$ 
which are strictly invariant by $f$, i.e., $\mathcal{K}(X, f)=\{K \subseteq X: f(K) \subseteq K$ and $K$ compact $\}$. The topological entropy for $f$ is defined as

$$
\operatorname{ent}(f)=\sup _{K}\left\{h_{d}\left(\left.f\right|_{K}\right): K \in \mathcal{K}(X, f)\right\},
$$

where $h_{d}\left(\left.f\right|_{K}\right)$ is the Bowen's notion from Definition 2.8.

Remark 2.10. If $\mathcal{K}(X, f)=\varnothing$, then $\operatorname{ent}(f)=0$ (see the proof of Theorem 2.1 (b) in [6]).

\subsection{Topological entropy of maps on topological spaces}

Liu et al. [30] proposed a new notion of topological entropy that generalizes to arbitrary topological dynamical systems (compactness, metrizability or axioms of separation not necessarily require) in 2009. Liu et al.'s notion is metric-independent for any metrizable spaces and coincide with Adler et al.'s notion when the topological space restricted to be compact (see Section 3). Most of the fundamental properties of topological entropy are either preserve or similar to the others. In this subsection, we denote $X$ to be an arbitrary topological space and $f: X \rightarrow X$ a continuous map.

Definition 2.11. Let $(X, f)$ be an arbitrary topological dynamical system. Let $\alpha$ be an open cover of $X$ and $F$ be an $f$-invariant nonempty compact subset of $X$. We denote $M_{F}(\alpha)$ as the smallest cardinality of all subcovers (for $F$ ) of $\alpha$. Since $F$ is compact, a subcover (for $F$ ) of $\alpha$ must be exist. Therefore, $M_{F}(\alpha)$ must be a positive integer. Finally, let $L_{F}(\alpha)=\log M_{F}(\alpha)$.

Using Definition 2.2, we can find the join of any open cover (for $F$ ) of $\alpha$. Similar to Adler et al.'s notion, we obtain the following lemma and its proof can be found in [30, Lemma 2.3].

Lemma 2.12. The limit $\lim _{n \rightarrow \infty} \frac{1}{n} L_{F}\left(\mathrm{~V}_{j=0}^{n-1} f^{-j}(\alpha)\right)$ exists.

Definition 2.13. $\mathcal{C}(X, f)$ is denoted as the collection of all $f$-invariant nonempty compact subsets of $X$, i.e., $\mathcal{C}(X, f)=\{F \subseteq X: F \neq \varnothing, F$ compact and $f(F) \subseteq F\}$.

If the space $X$ is compact, together with the fact that $f(X) \subseteq X$, then we have $X \in \mathcal{C}(X, f)$ which implies $\mathcal{C}(X, f) \neq \varnothing$. Note that $\mathcal{C}(X, f)$ could be empty when $X$ 
is not compact. For example, we have a translation map $T: \mathbb{R} \rightarrow \mathbb{R}$ defined by $T(x)=x+5$. Clearly, we can see that $\mathcal{C}(\mathbb{R}, T)=\varnothing$.

Definition 2.14. Let $(X, f)$ be a topological dynamical system. For $F \in \mathcal{C}(X, f)$ and $\alpha$ be any open cover of $X$, topological entropy of $f$ on $F$ relative to $\alpha$ is denoted as ent ${ }^{*}(f, \alpha, F)=\lim _{n \rightarrow \infty} \frac{1}{n} L_{F}\left(\mathrm{~V}_{j=0}^{n-1} f^{-j}(\alpha)\right)$. By taking the supremum over all open covers $\alpha$ of $X$ on ent $^{*}(f, \alpha, F)$, we get the topological entropy of $f$ on $F$ denoted by ent $^{*}(f, \alpha)$, i.e., ent ${ }^{*}(f, \alpha)=\sup _{\alpha}\left\{\operatorname{ent}^{*}(f, \alpha, F)\right\}$.

Definition 2.15. Let $(X, f)$ be a topological dynamical system. When $\mathcal{C}(X, f)$ $\neq \varnothing$, define

$$
\text { ent }^{*}(f)=\sup _{F}\left\{\operatorname{ent}^{*}(f, F): F \in \mathcal{C}(X, f)\right\}
$$

When $\mathcal{C}(X, f)=\varnothing$, define ent ${ }^{*}(f)=0$. ent ${ }^{*}(f)$ is said to be the topological entropy of $f$.

\section{Relations between Notions and Fundamental Properties of Topological Entropy}

In this section, we will give an overview on the relation between the notions in Section 2 and some fundamental properties of topological entropy.

\subsection{Relations between different notions of topological entropy}

Recall that for two metrics $d$ and $d^{\prime}$ on $X$, we say that they are uniformly equivalent if both identity functions $i d_{X}:(X, d) \rightarrow\left(X, d^{\prime}\right)$ and $i d_{X}:\left(X, d^{\prime}\right) \rightarrow(X, d)$ are uniformly continuous. In other words, $f \in U C(X, d)$ if and only if $f \in U C\left(X, d^{\prime}\right)$. If $X$ is compact and both metrics $d$ and $d^{\prime}$ are equivalent metrics, then they are uniformly equivalent. Any continuous map $f: X \rightarrow X$ is also uniformly continuous when $X$ is compact.

We know that Bowen's notion is defined on arbitrary metric space. As mentioned in [2], Bowen's notion is metric dependent. The following lemma and theorem show that if $X$ is compact, then Bowen's notion does not depend on the metric and it coincides with the classic Adler et al.'s notion. 
Lemma 3.1. [2] If $d$ and $d^{\prime}$ are uniformly equivalent and $f \in U C(X, d)$, then $h_{d}(f)=h_{d^{\prime}}(f)$.

Theorem 3.2. [51] If $X$ is a compact metrisable space and $d$ is any metric on $X$, then $h(f)=h_{d}(f)$.

Liu et al.'s notion is defined on arbitrary topological space. Recall that Adler et al. defined their notion on compact topological space. Hence, Liu et al.'s notion will coincide with Adler et al.'s notion in some special case, for example, when the topological space $X$ is compact. The result is shown in the next theorem and the proof of the theorem can refer to [30, Theorem 2.9].

Theorem 3.3. If $(X, f)$ is a compact topological dynamical system, then ent $^{*}(f)=h(f)$.

As mentioned in Section 2, Canovas and Rodriguez's notion is a modification from Bowen's notion. It is enough to study the relation between Canovas and Rodriguez's notion and Liu et al.'s notion. Since topological and metric spaces are not equivalent, Canovas and Rodriguez's notion and Liu et al.'s notion are essentially different in general. The next theorem shows the consistency between these two notions.

Theorem 3.4. [30] Let $(X, d)$ be a metric space and $f$ be a continuous mapping from $X$ into itself. Then the topological entropy according to Definition 2.15 and the topological entropy given by Definition 2.9 are consistent, i.e., $\operatorname{ent}^{*}(f)=\operatorname{ent}(f)$.

\subsection{Fundamental properties of topological entropy}

Topological entropy comes with some fundamental properties. We will discuss some fundamental properties of topological entropy based on Liu et al.'s notion. For other notions that we reviewed in Section 2, the fundamental properties of topological entropy will be similar or different due to the way they are being defined. Hence, for each fundamental property of topological entropy that we discuss, we will give a remark about the same fundamental property on other notions.

Recall that if we let $(X, f)$ and $(Y, g)$ be any topological dynamical systems and $\pi: X \rightarrow Y$ be a homeomorphism map such that $\pi \circ f=g \circ \pi$. Then $f$ is topologically conjugate to $g$ and map $\pi$ is known as conjugacy [50]. 
Theorem 3.5. [30] If $(X, f)$ and $(Y, g)$ are two topologically conjugated dynamical systems, then ent $^{*}(f)=$ ent $^{*}(g)$.

Remark 3.6. The notion given by Alder et al. [1], Bowen [2], Canovas and Rodriguez [6] are invariant of topological conjugacy as well.

Theorem 3.7. [30] Let $m$ be a positive integer, ent $^{*}\left(f^{m}\right) \geq m \cdot$ ent $^{*}(f)$. When $\mathcal{C}(X, f)=\mathcal{C}\left(X, f^{m}\right)$, then $\operatorname{ent}^{*}\left(f^{m}\right)=m \cdot \operatorname{ent}^{*}(f)$.

Remark 3.8. For Adler et al., Bowen, Canovas and Rodriguez's notions $[1,2,6]$, the topological entropy of $f^{m}$ is equal to $m$ times of the topological entropy of $f$.

For two topological dynamical systems $(X, f)$ and $(Y, g)$, we also can form the product space $X \times Y$ and a continuous map $f \times g: X \times Y \rightarrow X \times Y$ defined by $(f \times g)(x, y)=(f(x), g(y))$. The pair $(X \times Y, f \times g)$ forms a topological dynamical system.

Theorem 3.9. [30] Let $(X, f)$ and $(Y, g)$ be two topological dynamical systems, where $X$ and $Y$ are Hausdorff. Then ent ${ }^{*}(f \times g) \leq$ ent $^{*}(f)+$ ent $^{*}(g)$.

Remark 3.10. Adler et al. show that $h(f \times g)=h(f)+h(g)$ without the assumption that $X$ and $Y$ are Hausdorff [1, Theorem 3]. However Goodwyn [17] proved that the equality only holds if $X$ and $Y$ are both compact Hausdorff spaces. Hence for Adler et al.'s notion we will have the inequality $h(f \times g)=h(f)+h(g)$. Bowen's notion yields similar result to Theorem 3.9 (see [2] and [4]). Moreover, when one of the metric spaces is compact, then for Bowen's notion we obtain $h_{d}(f \times g)=$ $h_{d}(f)+h_{d}(g)$ [51, Theorem 7.10]. For Canovas and Rodriguez's notion, we will have the topological entropy of $f \times g$ is equal to the sum of the topological entropies of $f$ and $g$ [6].

Theorem 3.11. [30] Let $(X, f)$ be a topological dynamical system and assume that map $f$ is homeomorphism. If the conditions

(i) for any $F \in \mathcal{C}(X, f), f: F \rightarrow F$ is autohomeomorphism;

(ii) for any $F \in \mathcal{C}\left(X, f^{-1}\right), f^{-1}: F \rightarrow F$ is autohomeomorphism; holds, then ent ${ }^{*}(f)=\operatorname{ent}^{*}\left(f^{-1}\right)$. 
Remark 3.12. For a homeomorphism map $f$, the topological entropy of $f$ and the topological entropy of $f^{-1}$ are equal according to Adler et al. [1], Canovas and Rodriguez's notions [6]. For Bowen's notion, the topological entropy of $f$ and the topological entropy of $f^{-1}$ are not necessary equal under the condition $f$ is homeomorphism (see Remark 16 in [51]).

\section{Topological Entropy of Continuous Map for Induced Hyperspace}

The set valued dynamics that induced from original dynamics is known as induced hyperspace. We can find the topological entropy for the induced hyperspace in a similar fashion to the original dynamical system. Hence, there is a connection between the original system and the induced hyperspace. We discuss the formation of hyperspace and the connection of topological entropy between original and induced systems. Reader can refer famous sources such as [23], [34], [48] and [49] for further exploration about hyperspaces.

Definition 4.1. Let $(X, \tau)$ be a topological space. Then $2^{X}$ is defined as the family of all nonempty closed subsets of $X$. For any finite collection of sets $U_{1}, U_{2}, \ldots, U_{n} \in \tau$, let

$$
\left\langle U_{1}, U_{2}, \ldots, U_{n}\right\rangle=\left\{A \in 2^{X}: A \subset \bigcup_{i=1}^{n} U_{i} \text { and } A \cap U_{i} \neq \varnothing \text { for each } i=1, \ldots, n\right\} .
$$

The collection of all subsets of $2^{X}$ denoted as $\mathcal{B}=\left\{\left\langle U_{1}, U_{2}, \ldots, U_{n}\right\rangle: U_{i} \in \tau\right.$ for each $i=1, \ldots, n\}$ is a base for a topology of $2^{X}$ called the Vietoris topology or finite topology, $\tau_{v}$. We call the pair $\left(2^{X}, \tau_{v}\right)$ hyperspace of $X$.

Definition 4.2. When $(X, d)$ is a metric space, for any $A \in 2^{X}$ and $\varepsilon>0$, let $N(A, \varepsilon)=\{x \in X: d(x, a)<\varepsilon$ for some $a \in A\}$. We call $N(A, \varepsilon)$ as $\varepsilon$-neighborhood of $A$. For $A, B \in 2^{X}$, a map $H_{d}: 2^{X} \times 2^{X} \rightarrow[0, \infty)$ is defined as

$$
H_{d}(A, B)=\inf \{\varepsilon>0: A \subseteq N(B, \varepsilon) \text { and } B \subseteq N(A, \varepsilon)\} .
$$

$H_{d}$ (or simply denote as $H$ ) is a metric for $2^{X}$ known as the Hausdorff metric. 
Remark 4.3. From 0.13 of [49], we know that when $X$ is compact, the Vietoris topology and the topology induced by the Hausdorff metric are the same.

Definition 4.4. Let $f: X \rightarrow X$ be a self-continuous mapping of $X$. Then a continuous mapping $\bar{f}: 2^{X} \rightarrow 2^{X}$ given by $\bar{f}(A)=f(A)=\{f(a): a \in A\}$ for each $A \in 2^{X}$ is called as the map induced by $f$, or simply the induced map.

$\left(2^{X}, \bar{f}\right)$ is called as the induced hyperspace system induced by $(X, f)$. Canovas and Lopez [7] investigate the relation of topological entropy between the original system and the induced system using Bowen's notion. The result is shown in the following theorem.

Theorem 4.5. [7] Let $(X, d)$ be a compact metric space and let $f: X \rightarrow X$ be continuous. Let $\bar{f}: 2^{X} \rightarrow 2^{X}$ be the map defined above. Then $h_{d}(f) \leq h_{H}(\bar{f})$. Moreover, the inequality can be strict.

Remark 4.6. According to [30] or [38], if we use topological space instead of metric space, then we yield similar result for Theorem 4.5.

From Theorem 4.5, we can see that if the $(X, f)$ is topologically chaotic, then the $\left(2^{X}, \bar{f}\right)$ is topologically chaotic as well. However, Kwietniak and Oprocha [27] proved that the converse is false. The following examples taken from [27] show that if $h_{d}(f)=0$, then we can get either $h_{d}(\bar{f})=0$ or $h_{d}(\bar{f}) \geq 0$.

Example 4.7. Let $R_{\alpha}$ be an irrational rotation of a circle. Then $h_{d}\left(R_{\alpha}\right)=$ $h_{d}\left(\bar{R}_{\alpha}\right)=0$.

Example 4.8. Let $S$ be a subset of $\Sigma_{2}$ where symbol 1 only occurs at most once. Then for the subshift $\left(S, \sigma_{S}\right), h_{d}\left(\sigma_{S}\right)=0$ but $h_{d}\left(\bar{\sigma}_{S}\right)=\log 2$.

Remark 4.9. Reader can refer [32] for alternate proof of Example 4.8 and [28] if the map $f$ is homeomorphism.

\section{Conclusion and Recent Development}

We have explored some notions of topological entropy that are commonly seen in literature. We have also studied the relation between the notions and some fundamental 
properties of topological entropy. Furthermore, we have reviewed the connection of the topological entropy between the dynamical system and its induced system. To end this paper, we give a summary on the latest researches about topological entropy in dynamical systems.

One of the earliest results by Bowen [3] was a dynamical system with specification property must have positive entropy. In 2015, Dong [14] showed that there exist some dynamical systems satisfying almost specification property but with zero entropy, contradict with Bowen's result. Dong also proposed the sufficient and necessary condition for a system with almost specification property to have positive entropy. Kawan and Latushkin [22] derived the sufficient conditions for the variational principle that relate topological and measure-theoretic entropy together to hold for nonautonomous systems. In 2016, Zheng and Chen [42] defined Bowen entropy for amenable group action dynamical systems and showed that the Bowen entropy of the whole compact space for a given F $\phi$ lner sequence under tempered condition equal to the topological entropy of the systems.

Canovas, who did intensive researches in the topological entropy of fuzzified dynamical systems $[8,9,10]$ recently extended his study to a more general family of fuzzy sets on a compact interval, i.e., fuzzy sets with their level sets ( $\alpha$-cuts) have at most $m$ connected (convex) components, where $m$ represents some positive integer. Canovas and Kupka [11] proved that both the continuous interval map and its fuzzification to the space of fuzzy sets with the property above have positive topological entropy. Another interesting dynamics that has been work for a long time is the graph map, a compact connected one-dimensional branched manifold (see [31, 35, 41]). Recently Sun [40] generalised some equivalent conditions for an interval map has positive topological entropy to graph map.

Recently, researchers start to investigate the topological entropy of set-valued functions. In 2015, Carrasco-Olivera et al. [12] introduced two kinds of entropy which known as separated and spanning entropy for set valued functions. Furthermore they proved that these entropies satisfy some of the fundamental properties of the topological entropy of single-valued function. Later, Kelly and Tennant [24] introduced a new notion of topological entropy for set-valued function and showed that the topological entropy of the set-valued function and the topological entropy of the shift map on its orbit space are both equal. In 2016, Cordeiro and Pacifico [13] extended the notion of point-wise 
specification property and continuum-wise expansivity into set-valued function. They proved that set-valued function with point-wise specification property have positive entropy and gave sufficient conditions for a continuum-wise expansive set-valued function to have positive entropy. Meanwhile, Raines and Tennant [36] proved that the set-valued function with specification property will imply positive entropy and topological mixing.

One of the methods to study dynamical systems is through symbolic dynamics. In 2018, Li et al. [29] studied the topological entropy of the induced hyperspace dynamical systems of the symbolic dynamical systems and proved that entropy of the induced hyperspace dynamical systems of any symbolic dynamical system with two or more symbols is infinity.

\section{References}

[1] R. L. Adler, A. G. Konheim and M. H. McAndrew, Topological entropy, Trans. Amer. Math. Soc. 114 (1965), 309-319.

[2] R. Bowen, Entropy for group endomorphisms and homogeneous spaces, Trans. Amer. Math. Soc. 153 (1971), 401-414.

[3] R. Bowen, Periodic points and measures for Axiom A diffeomorphisms, Trans Amer. Math. Soc. 154 (1971), 377-397.

[4] R. Bowen, Erratum to "Entropy for group endomorphisms and homogeneous spaces", Trans. Amer. Math. Soc. 181 (1973), 509-510.

[5] R. Bowen, Topological entropy for noncompact sets, Trans. Amer. Math. Soc. 184 (1973), 125-136.

[6] J. S. Canovas and J. M. Rodriguez, Topological entropy of maps on the real line, Topology Appl. 153 (2005), 735-746.

[7] J. S. Canovas-Pena and G. S. Lopez, Topological entropy for induced hyperspace maps, Chaos Solitons Fractals 28 (2006), 979-982.

[8] J. S. Canovas and J. Kupka, Topological entropy of fuzzified dynamical systems, Fuzzy Sets and Systems 165 (2011), 67-79.

[9] J. S. Canovas and J. Kupka, On the topological entropy on the space of fuzzy numbers, Fuzzy Sets and Systems 257 (2014), 132-145.

[10] J. S. Canovas and J. Kupka, On fuzzy entropy and topological entropy of fuzzy extensions of dynamical systems, Fuzzy Sets and Systems 309 (2017), 115-130. 
[11] J. S. Canovas and J. Kupka, On topological entropy of Zadeh's Extension defined on piecewise convex fuzzy sets, in: Advances in Fuzzy Logic and Technology 2017, Cham: Springer, 2017, pp. 342-353.

[12] D. Carrasco-Olivera, R. M. Alvan, and C. A. M. Rojas, Topological entropy for setvalued maps, Discrete Contin. Dyn. Syst. Ser. B 20(10) (2015), 3461-3474.

[13] W. Cordeiro, and M. Pacifico, Continuum-wise expansiveness and specification for setvalued functions and topological entropy, Proc. Amer. Math. Soc. 144(10) (2016), 42614271 .

[14] Y. Dong, Systems with almost specification property may have zero entropy, Dyn. Syst. 31(2) (2016), 228-235.

[15] T. N. T. Goodman, Relating topological entropy and measure entropy, Bull. Lond. Math. Soc. 3 (1971), 176-180.

[16] L.W. Goodwyn, Topological entropy bounds measure-theoretic entropy, Proc Amer. Math. Soc. 23 (1969), 679-688.

[17] L.W. Goodwyn, The product theorem for topological entropy, Trans. Amer. Math. Soc. 158 (1971), 445-452.

[18] L.W. Goodwyn, Comparing topological entropy with measure-theoretic entropy, Amer. J. Math. 94 (1972), 366-388.

[19] M. Handel, B. Kitchens and D. J. Rudolph, Metrics and entropy for non-compact sets, Israel J. Math. 91 (1995), 253-271.

[20] J.E. Hofer, Topological entropy for noncompact spaces, Michigan Math. J. 21 (1974), 235-242.

[21] A. Katok, Fifty years of entropy in dynamics: 1958-2007, J. Mod. Dyn. 1(4) (2007), 545596.

[22] C. Kawan and Y. Latushkin, Some results on the entropy of non-autonomous dynamical systems, Dyn. Syst. 31(3) (2015), 251-279.

[23] J.L. Kelly, Hyperspaces of a continuum, Trans. Amer. Math. Soc. 52 (1942), 23-36.

[24] J. Kelly and T. Tennant, Topological entropy on set-valued functions, Houston J. Math. 43(1) (2017), 263-282.

[25] A. N. Kolmogorov, A new metric invariant of transient dynamical systems and automorphisms in Lebesgue spaces, Dokl. Akad. Nauk SSSR (N.S.) 119 (1958), 861-864.

[26] A. N. Kolmogorov, Entropy per unit time as a metric invariant of automorphisms, Dokl. Akad. Nauk SSSR 124 (1959), 754-755. 
[27] D. Kwietniak and P. Oprocha, Topological entropy and chaos for maps induced on hyperspaces, Chaos Solitons Fractals 33 (2007), 76-86.

[28] M. Lampart and P. Raith, Topological entropy for set valued maps, Nonlinear Anal. 73 (2016), 1533-1537.

[29] Z. Li, M. Wang and G. Wei, Induced hyperspace dynamical systems of symbolic dynamical systems, Int. J. Gen. Syst. 47 (2018), 809-820.

[30] L. Liu, Y. Wang and G. Wei, Topological entropy of continuous functions on topological spaces, Chaos Solitons Fractals 39 (2009), 417-427.

[31] J. Llibre and M. Misiurewicz, Horseshoes, entropy and periods for graph maps, Topology 21 (1993), 649-664.

[32] X. Ma, B. Hou and G. Liao, Chaos in hyperspace system, Chaos Solitons Fractals 40 (2009), 653-600.

[33] M. Malziri and M. R. Molaei, An extension of the notion of topological entropy, Chaos Solitons Fractals 36 (2008), 370-373.

[34] E. Michael, Topologies on spaces of subsets, Trans. Amer. Math. Soc. 71 (1951), 152$182,1951$.

[35] P. Oprocha and G. Zhang, On local aspects of topological weak mixing in dimension one and beyond, Studia Math. 202 (2011), 261-288.

[36] B. Raines and T. Tennant, The specification property on a set-valued map and its inverse limit, Houston J. Math. 44(2) (2018), 665-677.

[37] C. Shannon, A mathematical theory of communication, Bell System Tech. J. 27 (1948), 379-423, 623-656.

[38] P. Sharma and A. Nagar, Topological dynamics on hyperspaces, Appl. Gen. Topol. 11(1) (2010), pp. 1-19.

[39] Y. G. Sinai, On the concept of entropy for a dynamical system, Dokl. Akad. Nauk SSSR 124 (1959), 768-771.

[40] T. X. Sun, Topological entropy of a graph map, Acta Math. Sin. (Engl. Ser.) 34 (2018), 194-208.

[41] X. Ye, Non-wandering points and the depth of a graph, J. Aust. Math. Soc. 69A (2000), 143-152.

[42] D. Zheng and E. Chen, Bowen entropy for actions of amenable groups, Israel J. Math. 212(2) (2016), 895-911.

[43] L. Alseda, J. Llibre, and M. Misiurewicz, Combinatorial dynamics and entropy in dimension one, in: Advanced Series in Nonlinear Dynamics, Vol. 5, River Edge, NJ: World Scientific, 2000. 
[44] L. Block and W. A. Coppel, Dynamics in One Dimension, Lecture Notes in Math., Vol. 1523, Berlin: Springer, 1992.

[45] S. G. Brush, The Kinetic Theory of Gases: An Anthology of Classic Papers with Historical Commentary, Volume 1, History of Modern Physical Sciences, Imperial College Press, 2003.

[46] R. Clausius, The Mechanical Theory of Heat, London: Macmillan, 1879.

[47] T. Downarowicz, Entropy in Dynamical Systems, Vol. 18, Cambridge University Press, 2011.

[48] S. Macias, Topics on Continua, Pure Appl. Math., Vol. 275, Boca Raton, FL: Chapman \& Hall/CRC, 2005.

[49] S. B. Nadler, Jr., Hyperspaces of Sets, Monographs and Textbooks in Pure and Applied Math., Vol. 49, New York: Marcel Dekker, 1978.

[50] C. Robinson, Dynamical Systems: Stability, Symbolic Dynamics, and Chaos, 2nd ed., Florida: CRC Press, 1999.

[51] P. Walters, An Introduction to Ergodic Theory, New York: Springer-Verlag, 1982. 